\title{
Susan Palmer, The Nuwaubian Nation. Black Spirituality and State Control
}

Farnham, Ashgate, 2010, 177 p.

\section{Enzo Pace}

\section{OpenEdition}

\section{Journals}

Édition électronique

URL : http://journals.openedition.org/assr/22937

DOI : $10.4000 /$ assr.22937

ISSN : $1777-5825$

Éditeur

Éditions de l'EHESS

Édition imprimée

Date de publication : 31 décembre 2011

Pagination : 243

ISBN : 9782713223273

ISSN : 0335-5985

\section{Référence électronique}

Enzo Pace, «Susan Palmer, The Nuwaubian Nation. Black Spirituality and State Control», Archives de sciences sociales des religions [En ligne], 156 | octobre-décembre 2011, document 156-87, mis en ligne le 16 février 2012, consulté le 21 septembre 2020. URL : http://journals.openedition.org/assr/22937 ; DOI : https://doi.org/10.4000/assr.22937

Ce document a été généré automatiquement le 21 septembre 2020

(c) Archives de sciences sociales des religions 


\section{Susan Palmer, The Nuwaubian Nation. Black Spirituality and State Control}

Farnham, Ashgate, 2010, 177 p.

\section{Enzo Pace}

\section{RÉFÉRENCE}

Susan PALMER, The Nuwaubian Nation. Black Spirituality and State Control, Farnham, Ashgate, 2010, $177 \mathrm{p}$.

1 Susan Palmer n'en finit pas de nous surprendre. En 1994, lorsqu'elle se fit un nom comme experte des nouveaux mouvements religieux (je pense tout particulièrement à sa recherche remarquable et innovante sur le rôle des femmes dans son livre Moon Sisters, Krishna Mother, Rajneesh Lovers) et, encore en 2004, en réalisant une enquête - la première en termes d'étendue et de profondeur - sur le mouvement raëlien (Aliens Adored), S. Palmer avait déjà développé un sens inné pour les groupes religieux non conventionnels, de petites dimensions peut-être, mais significatifs lorsqu'il s'agit de comprendre les tensions internes au sein d'un milieu religieux déterminé.

2 C'est le cas de sa dernière recherche consacrée à un mouvement peu étudié par les sciences sociales des religions: le Nuwaubian (The Nuwaubian Nation). De ce mouvement-ci on trouve un écho dans la littérature américaine contemporaine, surtout du côté afro-musulman. C'est le cas, par exemple, de Knight.

Michael Muhammad Knight, auteur du livre Islampunk, qui a connu un grand succès aussi bien aux États-Unis qu'en Europe, devenant ainsi un culte pour les nouvelles générations musulmanes - et non musulmanes -, fait souvent référence dans ses récits aux diverses âmes de l'islam noir américain : il en parle avec ironie tout en réussissant en même temps à traduire, sous une forme littéraire, le niveau de différenciation sociale et religieuse qui caractérise une société aussi ouverte et complexe que peut 
l'être la société américaine. Passant de la Nation of Islam - le plus connu parmi les groupes en présence - au Moorish Science Temple, sans oublier le Fruit of Islam et le Five Percenters Nation de Clarence 13X (sur lequel M. Knight a écrit un livre très amusant: The Five Percenters: Islam, Hip Hop and the God's of New York, 2007), on a la nette impression d'une (petite) galaxie qui aurait explosé. Schismes, conflits entre leaders des différents mouvements, groupes qui changent de nom et parfois même de leader au cours de leur cycle vital, conflits récurrents avec la police et le FBI pour des affaires judiciaires ou fiscales très souvent infondées, mais parfois embrouillées et louches, font la une de l'histoire de l'islam noir d'Amérique. Knight fait justement une petite allusion sympathique aux Nuwaubians dans son dernier livre Journey to the End of Islam (New York, 2009).

S. Palmer situe donc efficacement l'analyse d'un mouvement spécifique - le Nuwaubian - fondé en 1967 par Dwight York à New-York City, sur le fond, changeant et hétéroclite, de l'islam noir, concentrant l'attention sur deux aspects spécifiques qui concernent le mouvement en question mais qui, en même temps, en transcendent les particularités.

Le premier aspect concerne la question suivante : quelle relation existe donc entre la recherche de l'identité noire parmi les Noirs d'Amérique et l'islam? Le second aspect, relié au premier, peut être résumé en ces termes : au sein de cette relation, comment l'islam a-t-il été réinterprété par les différents mouvements nés à l'intérieur de la communauté noire, quand les distances ont été prises par rapport à la tradition sunnite tout d'abord et lorsque l'on a tenté de recomposer la fracture entre sunnisme et chiisme? L'islam, en d'autres termes, semble une partition que les virtuoses de l'improvisation (leaders charismatiques comme l'ont été, par exemple, le fondateur de The Nuwaubian Nation ou encore Malcom X pour la Nation of Islam) réussissent à transformer en inventant de nouvelles tonalités et en arrivant même à créer de véritables dissonances qui parfois ne semblent avoir aucun lien avec l'islam traditionnel.

Le fait, comme dans le cas de Nuwaubian, qu'en 1993, York décide de vendre toutes ses propriétés, de quitter le quartier général de Brooklyn pour la Géorgie, et plus précisément Eatonton, où il achète un terrain de 475 acres qui servira à construire un village en style égyptien (appelé le Tama-Re), montre combien sa trajectoire de leader ne fut guère linéaire. Après un point de départ (1967) en référence au monde musulman (le premier nom qu'il donne au mouvement est justement Ansaar Pure Sufi, Les pures auxiliaires soufis), il finit (1968) par se faire appeler Imam Isa (Imam Jésus), une synthèse bizarre de l'islam, du christianisme et aussi de l'hébraïsme, quand il réinvente le mythe des tribus noires de Canaan desquelles descendraient les musulmans d'Amérique. En 1973, il se rebaptise Mahdi (un retour donc à la tradition afromessianique) et, en 1992, York renie une grande partie de l'islam, invoquant un retour aux pures racines monothéistes de l'hébraïsme. En construisant, finalement, le TamaRe en Géorgie, il réadmet les liens symboliques avec l'islam : le mouvement prendra désormais le nom d'United Nuwaubian Nation of Moors (abrégé en United Nuwaubian Nation) et Tama-Re devient dans l'imaginaire collectif du mouvement la Mecque de l'Occident pour les Noirs d'Amérique.

7 Palmer, non seulement reconstruit la figure du leader et la parabole du groupe religieux, jusqu'à l'incursion du FBI, en mai 2002, au temple de Tama-Re - signe d'une mauvaise réputation qu'entre temps le groupe s'était acquise dans une zone traditionnellement conservatrice de la Bible Belt, mais elle cherche aussi à argumenter 
une thèse qui réussisse à tenir unis deux thèmes: la reprise dans le mouvement de motifs propres à la tradition gnostique (thèse déjà connue, que les spécialistes du New Age ont depuis longtemps soutenue sur la base des affirmations des spécialistes du gnosticisme comme Rudolph ou Filoramo - qui n'est d'ailleurs pas cité - qui parlent de néo-gnosticisme) et la réapparition du thème du millénarisme qui, chez les Nuwaubians, s'accentue lors des répressions politique et judiciaire.

8 En réalité, S. Palmer explique que le millénarisme de York ne s'adresse pas au futur mais au passé, à la recherche d'un mythe originaire de l'identité spirituelle des Noirs d'Amérique. Pour cette raison, à travers une nuance difficile à traduire dans d'autres langues, Palmer parle d'une attitude millenarian (p.134) des Nuwaubians qui inventent un topos mental où ils imaginent qu'existent une terre, une foi, une culture originaires des Noirs d'Amérique. De ce point de vue, si l'hypothèse selon laquelle on peut interpréter le mouvement Nuwaubian comme l'une des nombreuses versions du néognosticisme moderne au sein d'une galaxie de spiritualité noire américaine apparaît convaincante, elle l'est déjà moins lorsque Palmer fait référence à l'esprit millénariste qui devrait le caractériser. Pas tant en raison du fait que techniquement, par millénarisme, on entend conventionnellement l'imagination du futur mais bien plutôt parce que le leader des Nuwaubians, même quand il se tourne vers un passé mythique, ne cesse d'utiliser des répertoires symboliques et des rhétoriques discursives cohérentes avec une vision millénariste : quand il imagine un temps où les angoisses du présent finiront et que les Noirs retrouveront finalement leur propre Terre (the Land), où tous les peuples de couleur "seront bienvenus et sauvés " (comme l'affirme un membre du mouvement interviewé par Palmer : p. 136).

Pour conclure, nous pouvons dire que cette recherche est seulement apparemment limitée à un mouvement relativement marginal dans le panorama socioreligieux américain. En réalité, le travail de Palmer peut être lu à la lumière de la théorie sociale dans le domaine religieux : la dés-institutionnalisation du phénomène religieux ouvre des espaces à la vertu de l'improvisation, aux si nombreux free-lance professionnels de l'esprit, en mesure de récupérer des symboles qui semblent hors circulation pour les réinterpréter, en inventant de nouveaux répertoires d'action sociale et de performances rituelles, greffant le tout sur l'histoire moderne et contemporaine des mouvements de libération des populations de couleur - pas seulement aux États-Unis, s'il est vrai que le phénomène néo-pentecôtiste en Afrique sub-saharienne reprend la lutte contre l'hérédité religieuse postcoloniale. 